\title{
Physics of a disordered Dirac point in epitaxial graphene from temperature-dependent magnetotransport measurements
}

\author{
J. Huang, ${ }^{1}$ J. A. Alexander-Webber, ${ }^{1}$ A. M. R. Baker, ${ }^{1}$ T. J. B. M. Janssen, ${ }^{2}$ A. Tzalenchuk,${ }^{2,3}$ V. Antonov, ${ }^{3}$ T. Yager, ${ }^{4}$ \\ S. Lara-Avila, ${ }^{4}$ S. Kubatkin, ${ }^{4}$ R. Yakimova, ${ }^{5}$ and R. J. Nicholas ${ }^{1, *}$ \\ ${ }^{1}$ Department of Physics, University of Oxford, Clarendon Laboratory, Parks Road, Oxford OX1 3PU, United Kingdom \\ ${ }^{2}$ National Physical Laboratory, Hampton Road, Teddington TW11 OLW, United Kingdom \\ ${ }^{3}$ Department of Physics, Royal Holloway, University of London, Egham TW20 0EX, United Kingdom \\ ${ }^{4}$ Department of Microtechnology and Nanoscience, Chalmers University of Technology, S-412 96 Göteborg, Sweden \\ ${ }^{5}$ Department of Physics, Chemistry and Biology (IFM), Linköping University, S-581 83 Linköping, Sweden
}

(Received 15 May 2015; published 6 August 2015)

\begin{abstract}
We report a study of disorder effects on epitaxial graphene in the vicinity of the Dirac point by magnetotransport. Hall effect measurements show that the carrier density increases quadratically with temperature, in good agreement with theoretical predictions which take into account intrinsic thermal excitation combined with electron-hole puddles induced by charged impurities. We deduce disorder strengths in the range 10.2-31.2 $\mathrm{meV}$, depending on the sample treatment. We investigate the scattering mechanisms and estimate the impurity density to be $3.0-9.1 \times 10^{10} \mathrm{~cm}^{-2}$ for our samples. A scattering asymmetry for electrons and holes is observed and is consistent with theoretical calculations for graphene on $\mathrm{SiC}$ substrates. We also show that the minimum conductivity increases with increasing disorder strength, in good agreement with quantum-mechanical numerical calculations.
\end{abstract}

DOI: 10.1103/PhysRevB.92.075407

PACS number(s): 72.80.Vp, 71.23.-k, 72.10.-d

\section{INTRODUCTION}

Many of the exceptional electronic properties of graphene arise from its linear dispersion relation [1,2]. However, when the Fermi energy approaches the Dirac point, its properties can be dominated by the effects of disorder, which can be both intrinsic (such as ripples and topological lattice defects) and extrinsic (including cracks/voids, adatoms, charged impurities, etc.), in general varying from sample to sample [3]. Of particular significance are the effects of disorder potentials on electrical transport properties [4] due to the lack of screening at very low carrier densities. Microscopically, the fluctuating electrostatic potential breaks up the intrinsically homogeneous charge distribution into electron-hole puddles [5-9]. This effect is recognized to mainly originate from unintendedly introduced charged impurities, whose type, spatial distribution, and density also depend on the sample environment, device fabrication techniques, and particularly graphene synthesis and treatment processes.

Recently, epitaxial graphene on $\mathrm{SiC}(\mathrm{SiC} / \mathrm{G})$ has been reported to have very high quantum Hall breakdown current density [10] which potentially allows a quantum electrical resistance standard operating at even higher temperatures and lower magnetic fields [11]. Low and well-controlled carrier density is required to achieve high breakdown current in these conditions, and understanding the disorder effects is therefore highly important. To date, there are very few experimental studies of disorder in epitaxial graphene grown on $\mathrm{SiC}$ due to the intrinsically high level of doping from the substrate [12]. In this paper, using extremely low carrier density epitaxial graphene, we describe the role of disorder in governing the temperature-dependent magnetotransport.

*r.nicholas@physics.ox.ac.uk

\section{METHODS AND METHODOLOGY}

Our $\mathrm{SiC} / \mathrm{G}$ samples were epitaxially grown on the $\mathrm{Si}$ terminated face of $4 \mathrm{H}-\mathrm{SiC}$ at $T=2000^{\circ} \mathrm{C}$ and $P=1 \mathrm{~atm}$ $\mathrm{Ar}$, as reported elsewhere [11,13-15]. The as-grown samples have large uniform monolayer areas, where devices with an eight-leg Hall bar geometry of various sizes were fabricated using standard electron-beam lithography followed by $\mathrm{O}_{2}$ plasma etching and large-area titanium-gold contacting. A nonvolatile polymer gating technique was used to control the carrier density in epitaxial graphene by room-temperature UV illumination [16] or corona discharge [17]. The polymer gates consist of bilayer polymer coating on top of the graphene Hall bars, forming SiC/graphene/polymer heterostructures. The first layer is PMMA/MMA copolymer, followed by the second layer of UV sensitive polymer ZEP520A [16]. Both $\mathrm{dc}$ and ac magnetotransport measurements were carried out using an Oxford Instruments $21 \mathrm{~T}$ superconducting magnet with a variable temperature insert which allows temperaturedependent measurements from 1.4 up to $300 \mathrm{~K}$.

Magnetotransport measurements were made on three SiC/G devices, which we denote CD1, CD2, and UV1. We used two different techniques to reduce the relatively high initial electron density and tune the Fermi level to the vicinity of the Dirac point, where four-probe resistance maxima were observed: $\mathrm{CD} 1$ and $\mathrm{CD} 2$ were treated with multiple negative ion projections onto the bilayer polymer gate, produced by corona discharge using a piezo-activated antistatic gun [17], resulting in extremely low final electron densities of 1.2 and $1.3 \times 10^{10} \mathrm{~cm}^{-2}$, respectively; UV1 was treated with deep UV illumination using a 248-nm mercury lamp [16] which eventually reduced the electron density to $8 \times 10^{10} \mathrm{~cm}^{-2}$. As we will show below, these values should not be treated as the real electron densities, but merely are effective carrier densities, $n_{\text {eff }}$, calculated from the low-field Hall coefficients at $1.4 \mathrm{~K}$ assuming a homogeneous landscape with a single type 

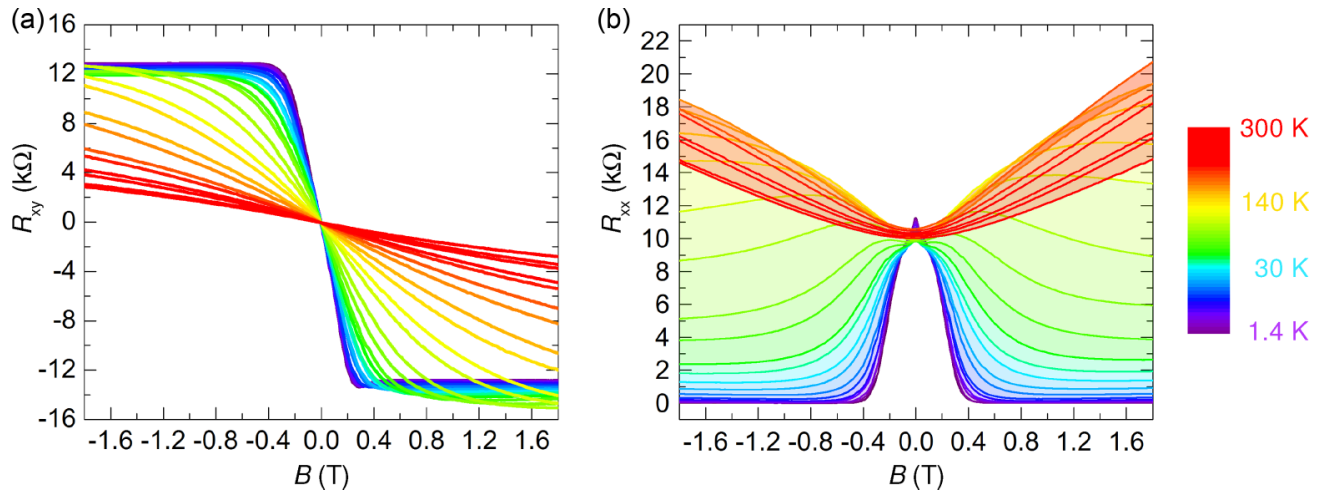

FIG. 1. (Color online) The Hall resistance $R_{x y}$ and the longitudinal resistance $R_{x x}$ as a function of magnetic field at temperatures from 1.4 to $300 \mathrm{~K}$ for sample CD2. The sample enters the quantum Hall regime already from about $0.6 \mathrm{~T}$ as observed from the quantized $R_{x y}$ and the vanishing $R_{x x}$ at low temperatures.

of charge carrier. In the absence of disorder, these densities would correspond to an upper limit for the Fermi energy $\left(E_{F}=\right.$ $\hbar v_{F} \sqrt{\pi n_{\text {eff }}}$, where $v_{F}$ is the Fermi velocity), which is between 12.7 and $32.9 \mathrm{meV}$, based on the assumption of a linear dispersion where the density of states vanishes at the Dirac point [18]. In reality, due to the effects of disorder, a residual density of states and coexistence of electrons and holes [6] at $E_{F} \rightarrow 0$ are expected, and thus the determination of an extremely low Fermi energy from Hall effect measurements becomes nontrivial. The overall net charge density is much lower than $n_{\text {eff }}$, but differences in the mobilities of the two carrier types still create a finite Hall coefficient at the Dirac point, which corresponds to the resistivity maximum studied here.

\section{RESULTS AND DISCUSSIONS}

\section{A. Intrinsic excitation in the presence of electron-hole puddles}

In Fig. 1, we present typical experimental results: the Hall resistance $R_{x y}$ and the longitudinal resistance $R_{x x}$ of sample CD2 as a function of magnetic field at temperatures from 1.4 to $300 \mathrm{~K}$. In our study, all three devices show similar behavior, as shown in Fig. 1. Due to the extremely low carrier densities of the samples, quantum Hall plateaux corresponding to the filling factor $v=2$ can be observed already from about $0.6 \mathrm{~T}$ at $1.4 \mathrm{~K}$. The Hall resistance becomes significantly nonlinear when approaching the quantum Hall regime. Therefore, to extract the zero-field carrier densities of our devices, only Hall coefficients between -0.1 and $+0.1 \mathrm{~T}$ are used.

It has been theoretically studied and experimentally confirmed that close to the Dirac point, as a consequence of disorder, the carrier density landscape is extremely inhomogeneous and electron-hole puddles form [4-9]. Classically, the low-field Hall coefficient in the presence of both electrons and holes is given by

$$
R_{H} \equiv \frac{E_{y}}{J_{x} B}=-\frac{1}{e} \frac{n_{e} \mu_{e}^{2}-n_{h} \mu_{h}^{2}}{\left(n_{e} \mu_{e}+n_{h} \mu_{h}\right)^{2}},
$$

where $n_{e}\left(n_{h}\right)$ and $\mu_{e}\left(\mu_{h}\right)$ are the electron (hole) density and mobility, respectively. Similar two-carrier analyses are also found in the literature for this electron-hole coexistence regime in monolayer and bilayer graphene [19,20]. The carrier density directly extracted from this two-carrier low-field Hall effect is, therefore, effectively,

$$
n_{\mathrm{eff}}=\frac{\left(n_{e} \mu_{e}+n_{h} \mu_{h}\right)^{2}}{n_{e} \mu_{e}^{2}-n_{h} \mu_{h}^{2}} .
$$

When the Fermi energy is zero, i.e., at charge neutrality point $(\mathrm{CNP}), n_{e}=n_{h}>0$. Thus, $n_{\mathrm{eff}}=\alpha n_{e}$, where $\alpha=$ $\frac{\frac{\mu_{e}}{\mu_{h}}+1}{\frac{\mu_{e}}{\mu_{h}}-1}$. Notably, for electronlike behavior $\left(R_{H}<0\right), \alpha>0$; for holelike behavior $\left(R_{H}>0\right), \alpha<0$.

We now analyze the temperature dependence of the effective carrier density $n_{\text {eff }}$, as shown in Fig. 2, for the three devices. A quadratic increase of $n_{\text {eff }}$ with increasing temperature can be clearly observed for all of the samples. Each sample also exhibits a distinct nonzero residual charge density at the low-temperature limit even when $E_{F} \rightarrow 0$, indicating that the potential landscape of our devices is highly inhomogeneous. These features are clearly different from the Arrhenius behavior of conventional semiconductors and intrinsic thermal activation in graphene when no disorder effects are accounted for (i.e., there is no residual carrier density). Accurate fitting

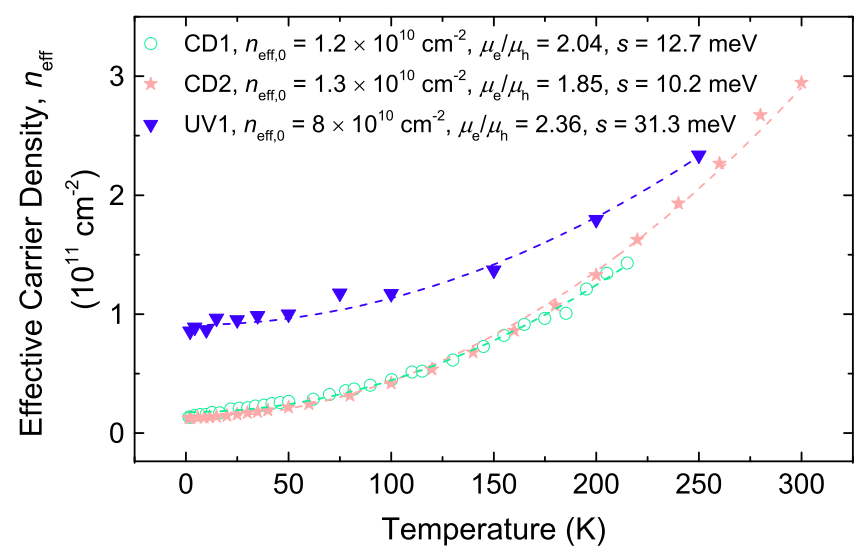

FIG. 2. (Color online) Temperature dependence of the effective carrier densities $n_{\text {eff }}$ deduced using Eqs. (1) and (2) for sample CD1, $\mathrm{CD} 2$, and UV1. Quadratic increase with increasing temperature is observed, together with nonvanishing carrier densities $n_{\text {eff, } 0}$ at $T \rightarrow 0$ $\mathrm{K}$. The experimental data is well fitted using Eqs. (4) and (5), as shown in the figure (dashed lines), where the disorder potential strength $s$ and the mobility ratio $\mu_{e} / \mu_{h}$ are extracted from the fitting. 
TABLE I. Energy fluctuations of electron-hole puddles in graphene. (Results of our analysis of magnetotransport data are highlighted in bold. KPM: Kelvin probe microscopy; SET: singleelectron transistor; STM: scanning tunneling microscopy; STS: scanning tunneling spectroscopy; CVD: chemical vapor deposition.)

Synthesis (treatment) Disorder strength (meV) Probing method

\begin{tabular}{lcc}
\hline Epitaxial on $\mathrm{SiC}(\mathbf{C D 1})$ & $\mathbf{1 2 . 7} \pm \mathbf{0 . 6}$ & Magnetotransport \\
Epitaxial on $\mathrm{SiC}(\mathbf{C D 2})$ & $\mathbf{1 0 . 2} \pm \mathbf{0 . 4}$ & Magnetotransport \\
Epitaxial on $\mathrm{SiC}(\mathbf{U V 1})$ & $\mathbf{3 1 . 3} \pm \mathbf{2 . 0}$ & Magnetotransport \\
Epitaxial on $\mathrm{SiC}(\mathbf{A O})$ & $\mathbf{1 5} \pm \mathbf{1}$ & Magnetotransport \\
Epitaxial on $\mathrm{SiC}$ & 12 & $\mathrm{KPM}[5]$ \\
Exfoliated on $\mathrm{SiO}_{2} / \mathrm{Si}$ & 50 & $\mathrm{SET}[6]$ \\
Exfoliated on $\mathrm{SiO}_{2} / \mathrm{Si}$ & $\sim 20$ & $\mathrm{STM}[7]$ \\
Exfoliated on $\mathrm{h}-\mathrm{BN}$ & 5.4 & STM [8] \\
CVD on $\mathrm{Ir}(111)$ & $\sim 30$ & STM/STS [9] \\
\hline
\end{tabular}

can be made based on the theory [4] assuming that the electronic potential energy of disordered graphene follows Gaussian statistics, which give the probability of finding the local potential within a range $d V$ about $V$,

$$
P(V) d V=\frac{1}{\sqrt{2 \pi s^{2}}} e^{-\frac{V^{2}}{2 s^{2}}} d V
$$

where $s$ is a parameter used to characterize the strength of the potential fluctuations. As a consequence, the temperaturedependent charge density at CNP for both electrons and holes is [4]

$$
n_{e}(T)=n_{h}(T)=\frac{g_{s} g_{v}}{2 \pi\left(\hbar v_{F}\right)^{2}}\left[\frac{s^{2}}{4}+\frac{\left(\pi k_{B} T\right)^{2}}{12}\right],
$$

where $g_{s}=g_{v}=2$ are the spin and valley degeneracies, and $v_{F} \approx 10^{6} \mathrm{~m} / \mathrm{s}$ is the Fermi velocity. The temperature dependence of the effective carrier density is, therefore,

$$
n_{\mathrm{eff}}(T)=\alpha n_{e}(T),
$$

where $\alpha$ is assumed to be constant over the temperature range under consideration. The predicted temperature dependence from Eqs. (4) and (5) fits the experimental data very well (Fig. 2), giving potential fluctuation strengths $s=12.7,10.2$, and $31.3 \mathrm{meV}$, and prefactors $\alpha$ which translate into mobility ratios of electrons to holes $\mu_{e} / \mu_{h}=2.04,1.85$, and 2.36 , for the devices CD1, CD2, and UV1, respectively. Table I shows comparisons of the potential fluctuations, due to electron-hole puddles, between the values deduced from our magnetotransport measurements and those found in the literature [5-9], where most of the characterizations are based on STM. Table I also includes the disorder strength $(15 \pm 1 \mathrm{meV})$ from our analysis of the published data for $\mathrm{SiC} / \mathrm{G}$ samples exposed to aqueous-ozone (AO) processing [21], which results in high mobility and extremely low $p$-type doping with an effective carrier density $n_{\mathrm{eff}, 0}=-4.0 \times 10^{10} \mathrm{~cm}^{-2}$ (negative sign for holelike behavior) from Hall measurements. We find that the disorder strengths measured in our samples are consistent with those reported previously for $\mathrm{SiC} / \mathrm{G}$, and are smaller than those of CVD and exfoliated samples on $\mathrm{SiO}_{2}$, while they are slightly larger than that of exfoliated graphene on h-BN, which is an atomically smooth, dangling-bond-free, and latticematched substrate to support high-quality graphene [22].
These comparisons suggest that $\mathrm{SiC} / \mathrm{G}$ generally has very good quality and relatively small amounts of disorder, even though the actual characteristics are expected to vary from sample to sample and may also be sensitive to the sample treatment, as seen from Table I. At the same time, it is demonstrated that magnetotransport measurement is an additional effective method to investigate the disorder effects and characteristics in graphene.

\section{B. Scattering mechanisms}

To evaluate the scattering mechanisms in our $\mathrm{SiC} / \mathrm{G}$ samples, we now turn to examine the temperature dependence of the longitudinal conductivity $\sigma_{x x}$ and the electron mobility, as shown in Fig. 3. Carrier mobilities of individual species are (a)

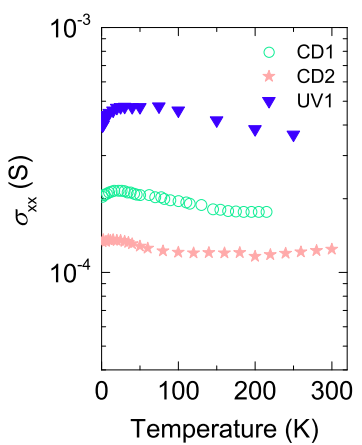

(c)

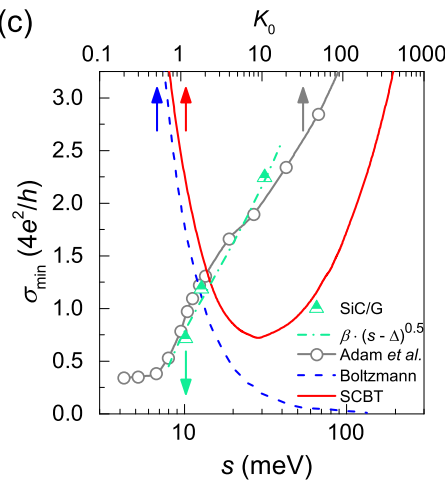

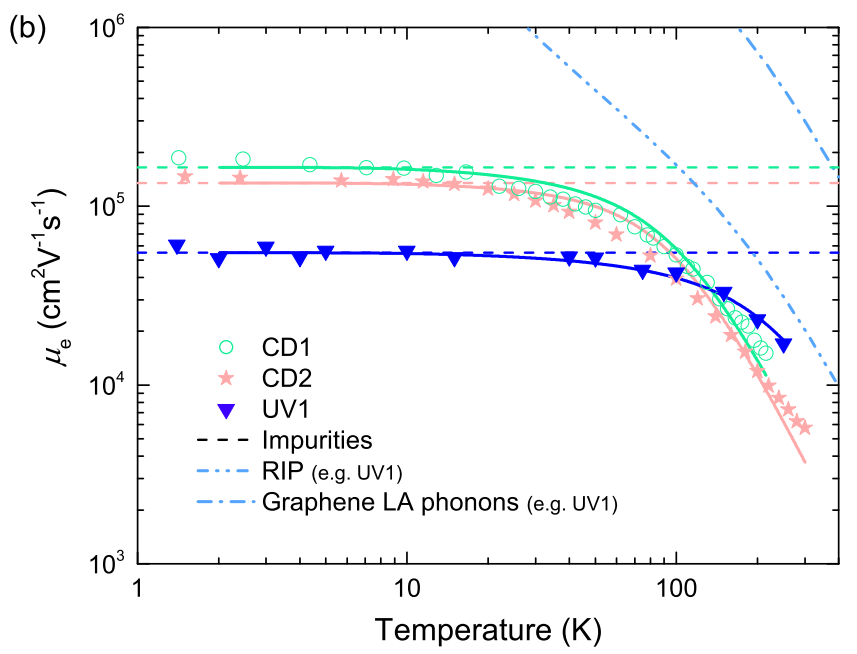

FIG. 3. (Color online) (a) The longitudinal conductivity as a function of temperature, where weak nonmonotonic dependences are shown. (b) The temperature dependence of the electron mobility of our samples. Individual contributions due to impurity scattering (green, pink, and blue dashed lines) for all three samples, LA phonon scattering (blue dash-dotted line), and RIP scattering (blue dash-double-dotted line) for UV1 as an example are shown. The solid lines represent the overall $\mu_{e}(T)$ dependence by fitting the experimental data. (c) $\sigma_{\min }$ as a function of disorder strength. An $\beta(s-\Delta)^{\frac{1}{2}}$ dependence (green dash-dotted line) is observed from our experimental data (green triangles). $\sigma_{\min }$ as a function of $K_{0}$ from numerical calculations by Adam et al. (gray circles and solid line), as well as predictions using the Boltzmann theory (blue dashed line) and the self-consistent Boltzmann theory (SCBT) (red solid line) are also shown [34]. 
calculated classically based on

$$
\sigma_{x x}=e\left(n_{e} \mu_{e}+n_{h} \mu_{h}\right)=\frac{e n_{\mathrm{eff}}}{\alpha}\left(\mu_{e}+\mu_{h}\right),
$$

via Eq. (5) and the value $\frac{\mu_{e}}{\mu_{h}}$ deduced from $\alpha$. It is observed that $\sigma_{x x}(T)$ remains slowly varying with weak nonmonotonic fluctuations for a large range of temperatures. Similar behavior has been reported for monolayer graphene samples when $E_{F} \approx 0[23,24]$, and this is clearly different from thermally activated conductivity in conventional gapped semiconductors and from phonon-limited behavior in graphene, which will result in a $T^{-4}$ or $T^{-1}$ dependence $[25,26]$ at low or high temperatures, respectively, due to intravalley acoustic phonon scattering. It should be pointed out that this temperature dependence of conductivity in our extremely low carrier density samples could be a combination of various contributions. It is believed that this weakly varying conductivity is mainly due to the temperature-dependent carrier density as described above and the $\mu(T)$ dependence as we will discuss below. At the lowest temperatures, there are also temperature-dependent weak localization corrections, which can be seen from Fig. 1(b) around $B=0 \mathrm{~T}$, but have been excluded in Fig. 3(a). Figure 3(b) shows the electron mobility as a function of temperature, as well as the mobility limits as a result of various scattering mechanisms, including impurity scattering, scattering by longitudinal acoustic (LA) phonons in graphene, and scattering by remote interfacial phonons (RIP) at the $\mathrm{SiC/graphene} \mathrm{interface}[27,28]$. In the case of charged impurities, carrier mobility is inversely proportional to the impurity density $n_{\text {imp }}[18,29]$,

$$
\mu_{\mathrm{imp}} \approx \frac{C_{0}}{n_{\mathrm{imp}}},
$$

where $C_{0}$ is a constant. For LA phonon scattering [25],

$$
\mu_{L A}=\frac{e \hbar \rho_{s} v_{s}^{2} v_{F}^{2}}{\pi n_{e} D_{A}^{2} k_{B} T},
$$

where $\rho_{s}=7.6 \times 10^{-7} \mathrm{~kg} / \mathrm{m}^{2}$ is the two-dimensional mass density, $v_{s}=1.7 \times 10^{4} \mathrm{~m} / \mathrm{s}$ is the sound velocity, and $D_{A}=$ $18 \mathrm{eV}$ is the acoustic deformation potential. The RIP limited mobility is given by $[27,28]$

$$
\mu_{R I P}=\frac{1}{n_{e} e}\left\{\sum_{i}\left[\frac{C_{i}}{\exp \left(\frac{E_{i}}{k_{B} T}\right)-1}\right]\right\}^{-1},
$$

where $C_{i}$ and $E_{i}$ are electron-phonon coupling constants and phonon energies, respectively, of the phonon modes under consideration. To fit our data, we first considered three phonon modes: two out-of-plane acoustic phonon modes in epitaxial graphene $\left(E_{1}=70 \mathrm{meV}\right.$ and $\left.E_{2}=16 \mathrm{meV}\right)[27,30]$ and a surface phonon mode of $4 \mathrm{H}-\mathrm{SiC}\left(E_{3}=117 \mathrm{meV}\right)[27,28,31]$. However, due to their relative large phonon energies, none of these can yield a reasonable fit, which can only be obtained [Fig. 3(b), solid lines] when an additional low-energy phonon mode $\left(E_{4} \approx 2 \mathrm{meV}\right.$ ) is introduced. This is consistent with the previously reported results [27,28,32,33], and this low-frequency remote phonon mode has been recognized to originate from the interaction between graphene and the buffer layer, that they are oscillating out-of-phase parallel to each other.
It can be seen from Fig. 3(b) that impurity scattering plays the most dominant role at low temperatures $(<100 \mathrm{~K})$, while the high-temperature mobility is probably limited by RIP scattering, since LA phonons make only a small contribution to the overall mobility for temperatures below $400 \mathrm{~K}$. Using $C_{0} \approx$ $5 \times 10^{15} \mathrm{~V}^{-1} \mathrm{~s}^{-1}$ [29], the densities of charged impurities for our $\mathrm{SiC} / \mathrm{G}$ samples are estimated to be $3.0-9.1 \times 10^{10} \mathrm{~cm}^{-2}$, which are 1-2 orders of magnitude lower than that in typical exfoliated [35] and CVD grown [36,37] graphene on $\mathrm{SiO}_{2}$, but are comparable to that of h-BN supported graphene [38], consistent with its high charge carrier mobility. Even though we restrict the above analysis to phonon and impurity scattering, other possible scattering mechanisms exist, such as scattering due to ripples [39,40] and very large defects [41]. Quantitative analysis of these mechanisms on our devices is rather difficult since systematic examination of the sample morphology is required and, on the other hand, the theoretical pictures are rather complicated and still contentious [42].

So far we have been able to identify that charge carrier scattering at low temperatures in our $\mathrm{SiC} / \mathrm{G}$ is mainly due to impurities, in the classical regime, where quantum corrections are suppressed by magnetic fields. It is these impurities which provide the same origin for generating the electronhole puddles at $E_{F} \rightarrow 0$. Furthermore, these impurities are most likely to be charged/Coulomb impurities rather than short-range impurities. The main evidence for this is the presence of unequal electron and hole mobilities, which is a consequence of the unbalanced scattering cross sections for charged scatterers in a system with two-dimensional relativistic dispersion $[29,43]$. This theory can be intuitively understood from the idea that an attractive potential scatters a charge carrier more effectively than a repulsive potential [43]. As presented in Fig. 2, we have obtained similar $\mu_{e} / \mu_{h}$ in the range of 1.85-2.36. According to the theory [43], assuming a single species of monovalent $(|Z|=1)$ impurities, the above mobility ratios can be translated into a dimensionless asymmetry factor $c=0.30-0.39$, which is used to characterize the strength of this asymmetry effect (i.e., $c=0$ for $\mu_{e}=\mu_{h}$ and $c \rightarrow 1$ for $\left.\mu_{e(h)} \gg \mu_{h(e)}\right)$. The nature of this asymmetry factor depends on the dielectric constant of the substrate: for $\mathrm{SiO}_{2},\left.c\right|_{\epsilon_{r}=3.9} \approx 0.46$; for $\mathrm{SiC}$, the same substrates as used in our devices, $\left.c\right|_{\epsilon_{r}=10.0} \approx 0.32$, which is in very good agreement with our experimental results. Small variations around the predicted value are expected, since the actual electrostatic environment of each $\mathrm{SiC} / \mathrm{G}$ sample could also be affected by the polymer top-gate dielectrics; meanwhile, the types and amounts of charged impurities present in our samples could be more complex.

\section{Minimum conductivity of disordered graphene}

Finally, the effects of disorder potential fluctuations on the low-temperature, nonvanishing minimum conductivity $\left(\sigma_{\min }\right)$ at the Dirac point are investigated for graphene in the diffusive transport regime. This property has been extensively considered theoretically and the two main existing approaches lead to contradictory results [34]. The semiclassical Boltzmann transport theory predicts a decreasing $\sigma_{\min }$ with increasing disorder strength. With a self-consistent modification to the Boltzmann theory, a subsequent increase of the minimum 
conductivity for higher disorder strengths is predicted. On the other hand, the minimum conductivity treated quantum mechanically [34,44-46] is increased for the entire disorder strength range for a noninteracting model using a Gaussian correlated disorder potential. Experimentally, very few studies can be found addressing this problem in the literature [29]. Shown in Fig. 3(c) is the minimum conductivity (at $B=0$ ) as a function of the disorder strength $s$ obtained from our measurements when quantum corrections have been taken into account, as well as theoretical predictions including the numerical calculation via the quantum-mechanical approach by Adam et al. [34], and results from the (self-consistent) Boltzmann theories, for $L=50 \xi$, where $L$ is the sample length, $\xi$ is the correlation length of the assumed random Gaussian potential $U(\mathbf{r})$ in the system, and the dimensionless parameter $K_{0} \propto\left\langle U(\mathbf{r}) U\left(\mathbf{r}^{\prime}\right)\right\rangle$ is the disorder strength used in the theories. Our experimental results show that the minimum conductivity increases with increasing $s$, roughly following a $\beta(s-\Delta)^{\frac{1}{2}}$ dependence locally in the $0.5-2.5 \times \frac{4 e^{2}}{h}$ range, highlighted by the green dash-dotted line in the figure, where $\beta$ and $\Delta$ are constants. This increase agrees qualitatively well with the theoretical predictions [34] from the quantummechanical approach, where we assume $s \propto \sqrt{K_{0}}$. However, our data do not agree with the results from the Boltzmann and the self-consistent Boltzmann theory, as shown in the figure. In addition, we note that the minimum conductivity may have a complex dependence on the sample length and details of quantum interference effects [34,47,48], and also be a function of the charged impurity density $n_{\text {imp }}$ indicated from previous experimental work by Chen et al. [29], whose results suggest that $\sigma_{\text {min }}$ drops with increasing $n_{\text {imp }}$ at low impurity densities and may saturate rapidly. To allow a more conclusive interpretation, however, more experimental data and systematic comparisons between well-controlled samples from different synthesis methods and a larger range of disorder potentials and impurity densities would be needed.

\section{CONCLUSIONS}

In summary, we have presented temperature-dependent magnetotransport measurements on epitaxial graphene. We have demonstrated the disorder effects when the Fermi energy lies in the vicinity of the Dirac point and have been able to identify the main origin of those effects to be charged impurities. The disorder strength and the impurity densities of our samples have been estimated from experimental results. We have also shown that the minimum conductivity increases with increasing disorder strength, in good agreement with numerical quantum-mechanical calculations. Overall, the application of this method can, therefore, provide an alternative and effective route for quantitatively studying the disorder characteristics in graphene and other two-dimensional materials.

\section{ACKNOWLEDGMENTS}

This work was partially supported by the U.K. EPSRC and NMS, E.U. Graphene Flagship (Contract No. CNECT-ICT604391), and EMRP GraphOhm. Work at Chalmers University of Technology was supported by the Swedish Foundation for Strategic Research (SSF), Linnaeus Centre for Quantum Engineering, Knut and Alice Wallenberg Foundation, and Chalmers AoA Nano. J.H. acknowledges financial support from the China Scholarship Council.
[1] P. R. Wallace, Phys. Rev. 71, 622 (1947).

[2] K. S. Novoselov, A. K. Geim, S. V. Morozov, D. Jiang, M. I. Katsnelson, I. V. Grigorieva, S. V. Dubonos, and A. A. Firsov, Nature (London) 438, 197 (2005).

[3] A. H. Castro Neto, F. Guinea, N. M. R. Peres, K. S. Novoselov, and A. K. Geim, Rev. Mod. Phys. 81, 109 (2009).

[4] Q. Li, E. H. Hwang, and S. Das Sarma, Phys. Rev. B 84, 115442 (2011).

[5] A. E. Curtin, M. S. Fuhrer, J. L. Tedesco, R. L. Myers-Ward, C. R. Eddy Jr., and D. K. Gaskill, Appl. Phys. Lett. 98, 243111 (2011).

[6] J. Martin, N. Akerman, G. Ulbricht, T. Lohmann, J. H. Smet, K. Von Klitzing, and A. Yacoby, Nat. Phys. 4, 144 (2008).

[7] Y. Zhang, V. W. Brar, C. Girit, A. Zettl, and M. F. Crommie, Nat. Phys. 5, 722 (2009).

[8] J. Xue, J. Sanchez-Yamagishi, D. Bulmash, P. Jacquod, A. Deshpande, K. Watanabe, T. Taniguchi, P. Jarillo-Herrero, and B. J. LeRoy, Nat. Mater. 10, 282 (2011).

[9] S. C. Martin, S. Samaddar, B. Sacépé, A. Kimouche, J. Coraux, F. Fuchs, B. Grévin, H. Courtois, and C. B. Winkelmann, Phys. Rev. B 91, 041406 (2015).

[10] J. A. Alexander-Webber, A. M. R. Baker, T. J. B. M. Janssen, A. Tzalenchuk, S. Lara-Avila, S. Kubatkin, R. Yakimova, B. A.
Piot, D. K. Maude, and R. J. Nicholas, Phys. Rev. Lett. 111, 096601 (2013).

[11] A. Tzalenchuk, S. Lara-Avila, A. Kalaboukhov, S. Paolillo, M. Syväjärvi, R. Yakimova, O. Kazakova, T. J. B. M. Janssen, V. Fal'ko, and S. Kubatkin, Nat. Nanotechnol. 5, 186 (2010).

[12] C. Riedl, C. Coletti, and U. Starke, J. Phys. D: Appl. Phys. 43, 374009 (2010).

[13] T. Yager, A. Lartsev, S. Mahashabde, S. Charpentier, D. Davidovikj, A. Danilov, R. Yakimova, V. Panchal, O. Kazakova, A. Tzalenchuk, S. Lara-Avila, and S. Kubatkin, Nano Lett. 13, 4217 (2013).

[14] T. J. B. M. Janssen, A. Tzalenchuk, R. Yakimova, S. Kubatkin, S. Lara-Avila, S. Kopylov, and V. I. Fal'ko, Phys. Rev. B 83, 233402 (2011).

[15] T. Yager, A. Lartsev, R. Yakimova, S. Lara-Avila, and S. Kubatkin, Carbon 87, 409 (2015).

[16] S. Lara-Avila, K. Moth-Poulsen, R. Yakimova, T. Bjørnholm, V. Fal'ko, A. Tzalenchuk, and S. Kubatkin, Adv. Mater. 23, 878 (2011).

[17] A. Lartsev, T. Yager, T. Bergsten, A. Tzalenchuk, T. J. B. M. Janssen, R. Yakimova, S. Lara-Avila, and S. Kubatkin, Appl. Phys. Lett. 105, 063106 (2014).

[18] E. H. Hwang, S. Adam, and S. Das Sarma, Phys. Rev. Lett. 98, 186806 (2007) 
[19] S. Wiedmann, H. J. van Elferen, E. V. Kurganova, M. I. Katsnelson, A. J. M. Giesbers, A. Veligura, B. J. van Wees, R. V. Gorbachev, K. S. Novoselov, J. C. Maan, and U. Zeitler, Phys. Rev. B 84, 115314 (2011).

[20] E. V. Kurganova, S. Wiedmann, A. J. M. Giesbers, R. V. Gorbachev, K. S. Novoselov, M. I. Katsnelson, T. Tudorovskiy, J. C. Maan, and U. Zeitler, Phys. Rev. B 87, 085447 (2013).

[21] T. Yager, M. J. Webb, H. Grennberg, R. Yakimova, S. Lara-Avila, and S. Kubatkin, Appl. Phys. Lett. 106, 063503 (2015).

[22] C. R. Dean, A. F. Young, I. Meric, C. Lee, L. Wang, S. Sorgenfrei, K. Watanabe, T. Taniguchi, P. Kim, K. L. Shepard, and J. Hone, Nat. Nanotechnol. 5, 722 (2010).

[23] K. I. Bolotin, K. J. Sikes, J. Hone, H. L. Stormer, and P. Kim, Phys. Rev. Lett. 101, 096802 (2008).

[24] S. V. Morozov, K. S. Novoselov, M. I. Katsnelson, F. Schedin, D. C. Elias, J. A. Jaszczak, and A. K. Geim, Phys. Rev. Lett. 100, 016602 (2008).

[25] E. H. Hwang and S. Das Sarma, Phys. Rev. B 77, 115449 (2008).

[26] T. Stauber, N. M. R. Peres, and F. Guinea, Phys. Rev. B 76, 205423 (2007).

[27] A. J. M. Giesbers, P. Procházka, and C. F. J. Flipse, Phys. Rev. B 87, 195405 (2013).

[28] S. Tanabe, Y. Sekine, H. Kageshima, M. Nagase, and H. Hibino, Phys. Rev. B 84, 115458 (2011).

[29] J.-H. Chen, C. Jang, S. Adam, M. S. Fuhrer, E. D. Williams, and M. Ishigami, Nat. Phys. 4, 377 (2008).

[30] J. Červenka, K. van de Ruit, and C. F. J. Flipse, Phys. Status Solidi B 247, 2992 (2010).

[31] H. Nienhaus, T. U. Kampen, and W. Mönch, Surf. Sci. Lett. 324, L328 (1995).

[32] C. Yu, J. Li, Q. B. Liu, S. B. Dun, Z. Z. He, X. W. Zhang, S. J. Cai, and Z. H. Feng, Appl. Phys. Lett. 102, 013107 (2013).
[33] N. Ray, S. Shallcross, S. Hensel, and O. Pankratov, Phys. Rev. B 86, 125426 (2012).

[34] S. Adam, P. W. Brouwer, and S. Das Sarma, Phys. Rev. B 79, 201404 (2009).

[35] Y.-W. Tan, Y. Zhang, K. Bolotin, Y. Zhao, S. Adam, E. H. Hwang, S. Das Sarma, H. L. Stormer, and P. Kim, Phys. Rev. Lett. 99, 246803 (2007).

[36] J. Lee, L. Tao, Y. Hao, R. S. Ruoff, and D. Akinwande, Appl. Phys. Lett. 100, 152104 (2012).

[37] J. Chan, A. Venugopal, A. Pirkle, S. McDonnell, D. Hinojos, C. W. Magnuson, R. S. Ruoff, L. Colombo, R. M. Wallace, and E. M. Vogel, ACS Nano 6, 3224 (2012).

[38] K. M. Burson, W. G. Cullen, S. Adam, C. R. Dean, K. Watanabe, T. Taniguchi, P. Kim, and M. S. Fuhrer, Nano Lett. 13, 3576 (2013).

[39] F. Guinea, J. Low Temp. Phys. 153, 359 (2008).

[40] M. I. Katsnelson and A. K. Geim, Phil. Trans. R. Soc. A 366, 195 (2008).

[41] J.-H. Chen, W. G. Cullen, C. Jang, M. S. Fuhrer, and E. D. Williams, Phys. Rev. Lett. 102, 236805 (2009).

[42] S. Das Sarma, S. Adam, E. H. Hwang, and E. Rossi, Rev. Mod. Phys. 83, 407 (2011).

[43] D. S. Novikov, Appl. Phys. Lett. 91, 102102 (2007).

[44] H. Suzuura and T. Ando, Phys. Rev. Lett. 89, 266603 (2002).

[45] A. Schuessler, P. M. Ostrovsky, I. V. Gornyi, and A. D. Mirlin, Phys. Rev. B 79, 075405 (2009).

[46] J. Tworzydło, C. W. Groth, and C. W. J. Beenakker, Phys. Rev. B 78, 235438 (2008).

[47] F. Ortmann, A. Cresti, G. Montambaux, and S. Roche, Europhys. Lett. 94, 47006 (2011).

[48] N. Leconte, A. Lherbier, F. Varchon, P. Ordejon, S. Roche, and J.-C. Charlier, Phys. Rev. B 84, 235420 (2011). 